\title{
Structure and Organisation of Interphase Microtubule Arrays: FESEM Provides a MAP
}

\author{
D.A. Barton, * R.L. Overall* \\ * School of Biological Sciences, Macleay Building A12, University of Sydney NSW 2006 \\ Australia
}

In plant cells at interphase, microtubules form a complex array that extends over the inner surface of the plasma membrane. They are involved in the directed growth of cellulose microfibrils within the cell wall as well as acting as tracks for the intracellular movement of cellular components. Microtubules were first discovered in the cortical regions of plant cells using transmission electron microscopy [1], and since then a number of different techniques have been developed to image and investigate the organisation and dynamics of microtubule arrays within both live and fixed cells. However, the precise organisation of individual microtubules within interphase arrays and their interaction with microtubule associated proteins (MAPs) still remains unclear. We developed a novel method to preserve interphase microtubule arrays within elongating leaf epidermal cells of Tradescantia virginiana, for examination with both confocal microscopy and field emission scanning electron microscopy (FESEM). Using FESEM, individual microtubules were easily resolved and two spatially distinct subpopulations of microtubules were identified within the arrays (Fig 1). Bundled microtubules lay adjacent to the plasma membrane, were located within $25 \mathrm{~nm}$ of their neighbours, were interlinked by crossbridges and were highly aligned within the main axis of each array. Discordant microtubules, lying deeper in the cytoplasm, crossed the paths of the microtubules lying adjacent to the plasma membrane. Discordant microtubules were randomly aligned throughout the arrays. When the array of a single cell was examined with both confocal microscopy and FESEM, individual microtubules, and in particular discordant microtubules resolved with electron microscopy were often not visible in the confocal image (Fig 2). The dynamics of the two microtubule subpopulations were investigated using the microtubule stabilising drug, taxol, and the microtubule depolymerising drug, oryzalin. Taxol treatment promoted microtubule co-alignment by increasing bundling and decreasing the number of discordant microtubules in the array. In arrays partially depolymerised by treatment with oryzalin, discordant microtubules were rarely found, and highly aligned, long, single microtubules lay on the plasma membrane. Discordant microtubules were concluded to be the more dynamic subpopulation of microtubules within an interphase array and bundled microtubules the more stable subpopulation. Co-localisation of specific antibodies against MAPs to microtubules was investigated using confocal microscopy and FESEM. On occasion no co-localisation was seen using immunofluorescence combined with confocal microscopy, but specific colocalisations were observed using FESEM. This work demonstrates the fundamental importance of investigating antibody localisations at high resolution to gain accurate insights into the intricate relationship between microtubules and their associated proteins.

[1] M.C. Ledbetter and K.R. Porter, J. Cell Biol. 19 (1963) 239-250

[2] This work was supported by an Australian Research Council Grant (A19905246) to RLO and an Australian Postgraduate Research Award to DAB. 


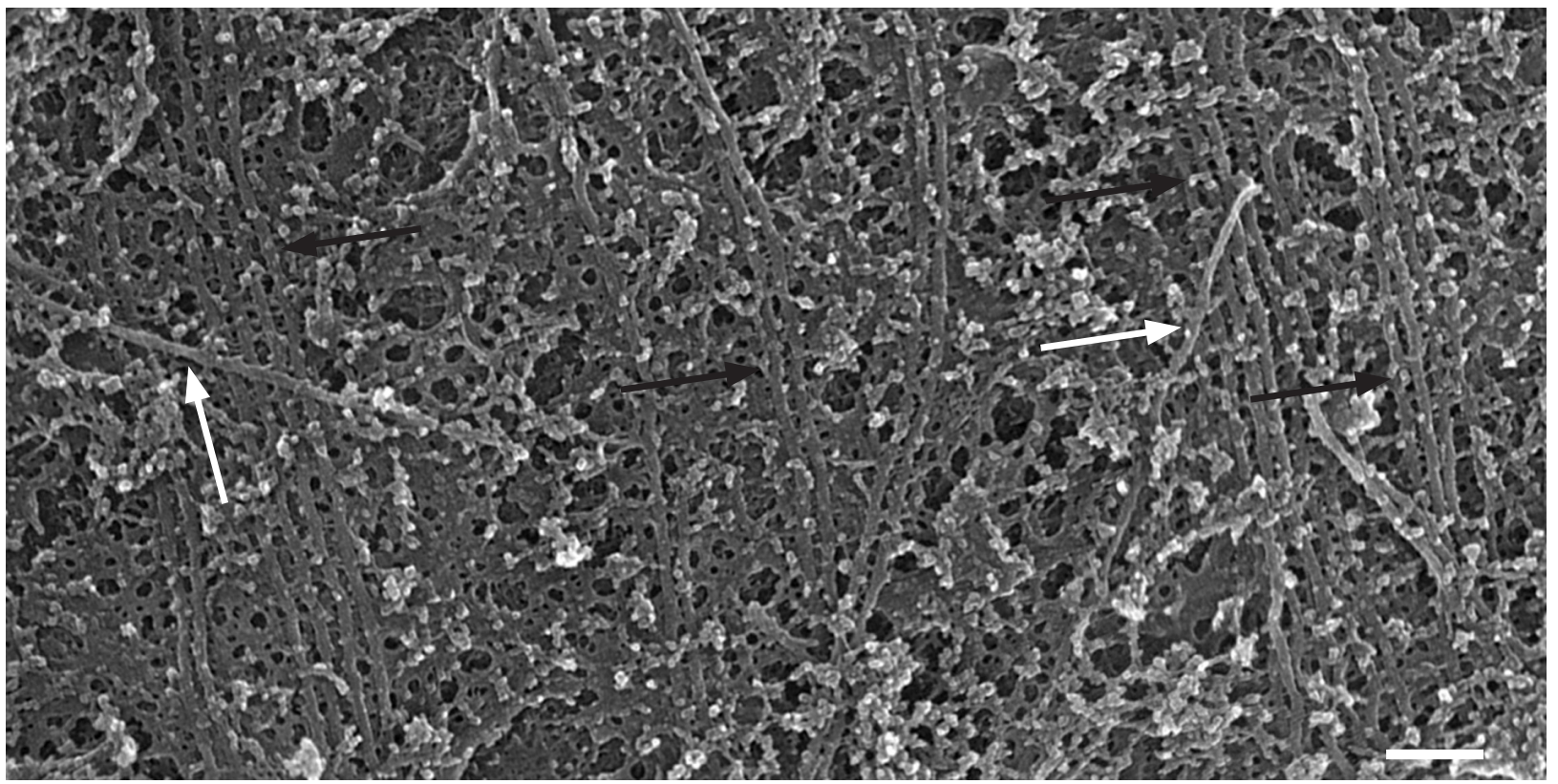

Fig 1. The two

microtubule subpopulations within interphase arrays: bundled microtubules (black arrows) against the plasma membrane and discordant microtubules (white arrows) lying deeper into the cytoplasm. Scale bar $=200 \mathrm{~nm}$

Fig 2. A microtubule array immunolabelled and imaged with a) confocal microscopy and b) FESEM. c) Individual microtubules within the boxed regions in b) and d). d) The position of the microtubules in the FESEM image was traced and laid over the correpsonding region in the confocal image (box in a). Individual microtubules sometimes corresponded to fluorescent lines (black arrows) but often were difficult to distinguish from background fluorescence (white arrows). Scale bars $\mathrm{a}, \mathrm{b}=5 \mu \mathrm{m}, \mathrm{c}=$ $200 \mathrm{~nm}, \mathrm{~d}=1 \mu \mathrm{m}$.
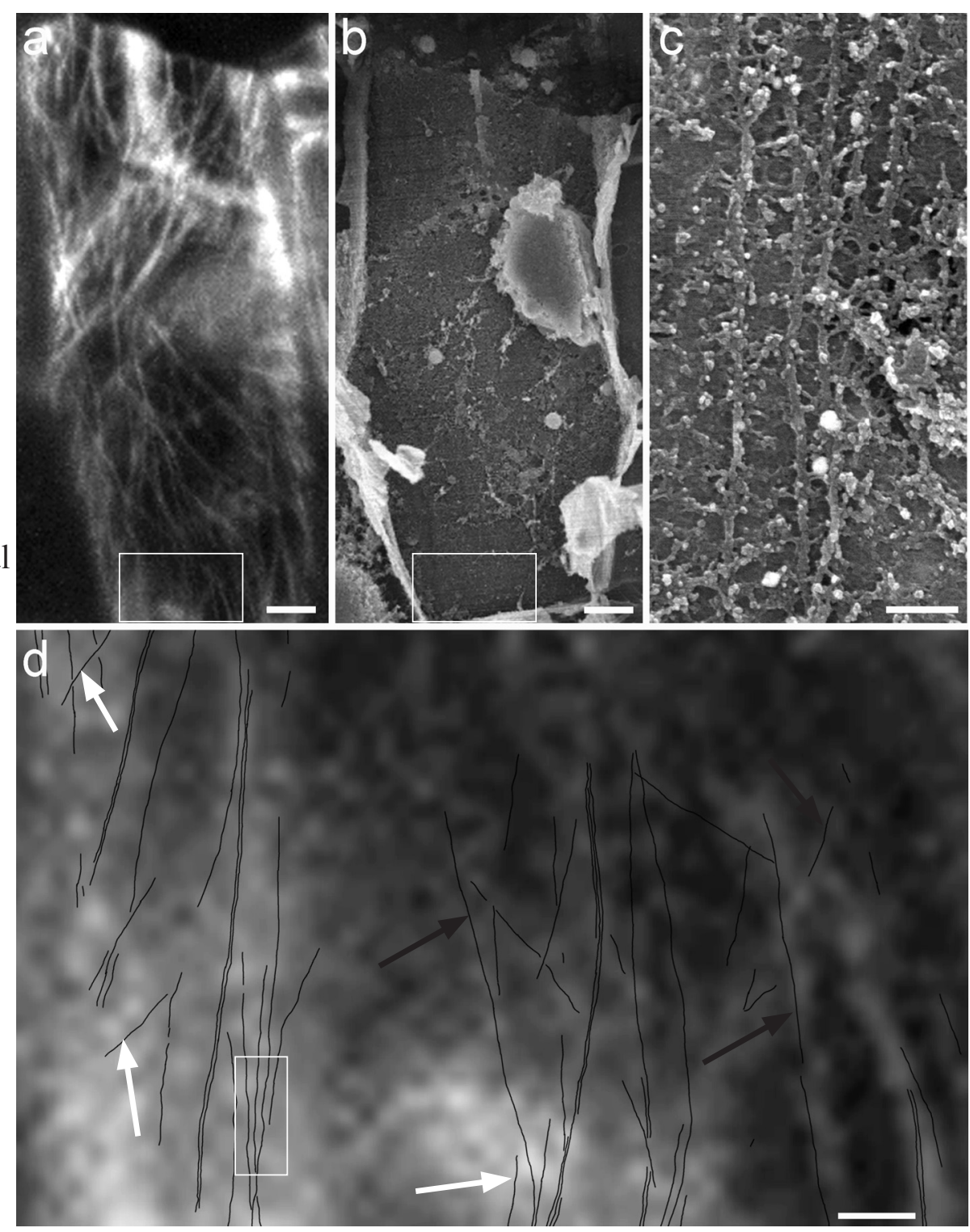